\title{
Progress and First Results with the New Multi- Frequency ECRH System for ASDEX Upgrade
}

\author{
Dietmar Wagner, Member, IEEE, Jörg Stober, Fritz Leuterer, George Sips, Gerhard Grünwald, \\ Francesco Monaco, Max Münich, Emanuele Poli, Harald Schütz, Francesco Volpe, Wolfgang \\ Treutterer, Hartmut Zohm, Thomas Franke, Manfred Thumm, Fellow, IEEE, Roland Heidinger, Gerd \\ Gantenbein, Andreas Meier, Walter Kasparek, Carsten Lechte, Alexander Litvak, Gregory Denisov, \\ Alexei Chirkov, Evgeny Tai, Leonid Popov, Vadim Nichiporenko, Vadim Myasnikov, Elena \\ Solyanova, Sergey Malygin and ASDEX Upgrade team
}

\begin{abstract}
A multi-frequency ECRH system is currently under construction at the ASDEX Upgrade tokamak experiment. The system employs depressed collector gyrotrons, step-tunable in the range 105-140 GHz, with a maximum output power of $1 \mathrm{MW}$ and a pulse length of 10s. One two-frequency GYCOM gyrotron is in routine operation at ASDEX Upgrade since 2006. A further extension of the system with 3 more gyrotrons is underway. An in-situ calibration scheme for the broad-band torus window has been developed. The system is equipped with fast steerable mirrors for real-time MHD control. The gyrotron and the mirrors are fully integrated into the discharge control system. The ECRH system turned out to be essential for the operation of H-modes after covering the plasma facing components of ASDEX Upgrade with tungsten. Deposition of ECRH inside $\rho_{\text {tor }}<0.2$ is necessary to prevent accumulation of $W$ in plasmas with high pedestal temperatures. With respect to the limited loop voltage available in ITER, the use of ECRH for neutral-gas preionization to facilitate plasma break-down and its application during the current ramp-up to increase the conductivity in order to save transformer flux have been demonstrated successfully for $105 \mathrm{GHz}, 3.2 \mathrm{~T}$ (O1-mode) and $140 \mathrm{GHz}, 2.2 \mathrm{~T}$ (X2-mode), corresponding to $170 \mathrm{GHz}$ at ITER with the full and half value of its foreseen toroidal field of $5.3 \mathrm{~T}$.
\end{abstract}

Index Terms-Electron Cyclotron Resonance Heating, Steptunable Gyrotron, Fast Steerable Launcher.

Manuscript received December 18, 2008.

D. Wagner, J. Stober, F. Leuterer, G. Sips, G. Grünwald, F. Monaco, M Münich, J. Stober, W. Treutterer, F. Volpe, H. Zohm and T. Franke are with the Max-Planck-Institut für Plasmaphysik, EURATOM-IPP, Boltzmansstr.2, D-85748 Garching, Germany (phone: 89-3299-2597; fax: 89-3299-1313; email: dietmar.wagner@ipp.mpg.de).

M. Thumm and G. Gantenbein are with the Forschungszentrum Karlsruhe, EURATOM-FZK, Institut für Hochleistungsimpuls- und Mikrowellentechnik, D-76021, Karlsruhe, Germany

R. Heidinger and A. Meier are with the Forschungszentrum Karlsruhe, EURATOM-FZK, Institut für Institut für Materialforschung, D-76021, Karlsruhe, Germany

W. Kasparek and C. Lechte are with the Institut für Plasmaforschung, Universität Stuttgart, D-70569 Stuttgart, Germany

A. Litvak, G.G. Denisov and A.V. Chirkov, are with the Institute of Applied Physics, RAS, 603600 Nizhny Novgorod, Russia

E.M. Tai, L.G. Popov, V.O. Nichiporenko, V.E. Myasnikov, E.A. Solyanova and S.A. Malygin are with GYCOM Ltd. 46 Ulyanov St., Nizhny Novgorod, Russia

\section{INTRODUCTION}

A single frequency ECRH system at $140 \mathrm{GHz}$ is in operation at the ASDEX Upgrade tokamak since 1996 [1]. The system consists of 4 gyrotrons with an output power of $500 \mathrm{~kW}$ each and a pulse length of 2 seconds. A new ECRH system is currently under construction that will in its final stage deliver a total power of $4 \mathrm{MW}$ with a pulse length of $10 \mathrm{~s}$ corresponding to the maximum flat top time of ASDEX Upgrade discharges [2]. An additional feature of the four gyrotrons of the new system is their frequency step tunability allowing operation at both 105 and $140 \mathrm{GHz}$, corresponding to the resonant frequency of a single disc CVD diamond window [3]. This capability extends the operating space of the ECRH at ASDEX in terms of both magnetic field and deposition radius [4]. A first two-frequency gyrotron is in routine operation since 2006. Its output power is $910 \mathrm{~kW}$ at $140 \mathrm{GHz}$ with a beam voltage of $U_{c}=-56 \mathrm{kV}$, a body voltage of $+29 \mathrm{kV}$ and a beam current of $\mathrm{I}_{\mathrm{b}}=37 \mathrm{~A}$. At $105 \mathrm{GHz}, 620 \mathrm{~kW}$ were achieved with $\mathrm{U}_{\mathrm{c}}=-49 \mathrm{kV}, \mathrm{U}_{\mathrm{b}}=+26 \mathrm{kV}$ and $\mathrm{I}_{\mathrm{b}}=34 \mathrm{~A}$. The transmission losses at both frequencies are below $10 \%$ which is in good agreement with theoretical predictions. They were measured using identical calorimetric loads installed both in the MOU and next to the torus window. There is no problem with arcing in the non-evacuated waveguide transmission line. Arcing occasionally occurred in the mirror box due to dust particles on the mirror surfaces. The gyrotron can be tuned to another frequency within approximately 20 minutes which corresponds to the time between two ASDEX Upgrade discharges. The broadband gyrotron output window still requires further development. Therefore the next two gyrotrons will also be two-frequency gyrotrons. The fourth gyrotron is planned to be a multi-frequency gyrotron with additional frequencies in between 105 and $140 \mathrm{GHz}$. For this gyrotron broadband vacuum windows are required both at the gyrotron and at the torus. The launchers of the new system have a poloidal fast steering capability that will allow for a change of the deposition location during the discharge without changing the toroidal magnetic field. The ultimate goal is to have a very flexible system for localized plasma heating and current drive 
that allows for feedback control of neoclassical tearing modes, pressure profile and transport [5].

\section{MULTI-FREQUENCY HARDWARE EXTENSIONS}

The transmission of the millimeter waves from the gyrotrons to the torus is mainly through non-evacuated corrugated waveguides with an inner diameter of $87 \mathrm{~mm}$. The total length of the transmission line is approximately $70 \mathrm{~m}$. These waveguides support the low-loss $\mathrm{HE}_{11}$ mode over the whole frequency band of the system. Each gyrotron is connected to a quasi-optical matching optics unit (MOU) that conditions the millimeter wave beam such that it can be effectively coupled to the corrugated waveguide. An individual set of two phase correcting mirrors is required for each frequency of a steptunable gyrotron to convert the gyrotron beam to a fundamental Gaussian beam that guaratees optimum coupling to the $\mathrm{HE}_{11}$ mode in the corrugated waveguide. These mirrors also correct the angular mismatch of up to $2^{\circ}$ of the different output beams with respect to the optical axis of the MOU and the waveguide input. This angular variation happens only in the plane perpendicular to the gyrotron axis and is due to the different caustic radii of the resonant gyrotron modes. The required space for these mirrors limits the number of applicable frequencies to four. The planned frequencies are 105, 117, 127 and $140 \mathrm{GHz}$, corresponding to resonant modes of the gyrotron. The phase correcting mirrors are mounted on turntables and are automatically set when the frequency is changed (Fig.1).
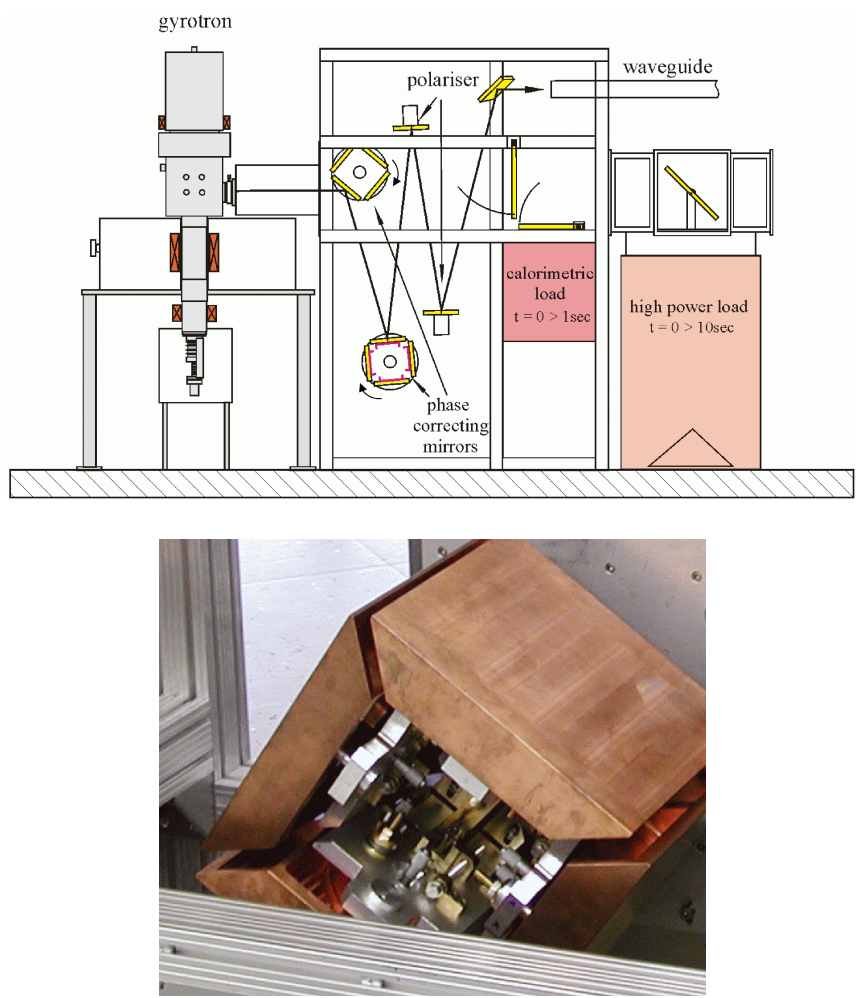

Fig. 1. MOU with phase correcting mirrors for the multifrequency gyrotron mounted on turntables.
The MOU also contains a pair of broadband polarizer mirrors [6] as well as a short-pulse load for a maximum pulse length of 1s. Additionally a long-pulse load capable of $1 \mathrm{MW}, 10 \mathrm{~s}$ can be coupled to each gyrotron beam successively. The gyrotron beam is linearly polarized and thus a broadband Brewster window is possible which is under development. The torus window however has to be transparent for an arbitrary elliptical polarization. Therefore a tunable double disc window was developed and constructed [7]. It takes advantage of FabryPerot reflection minima at frequencies where the single discs are not transparent. The reflectivity of the double disc window was measured and compared to theory (Fig.2). The inter-space between the two discs is evacuated to prevent arcing due to the field enhancement. The evacuation also leads to a slight bending of the discs which is responsible for the frequency shift of the measurement compared to theory (Fig.2). The measurement was repeated after demounting the disc as well as after bake-out up to $150^{\circ} \mathrm{C}$. To test an in-situ measurement method for the installed double-disc torus window, the beam was launched through $3 \mathrm{~m}$ of straight waveguide plus one miter bend. Again, this measurement gave the same result.

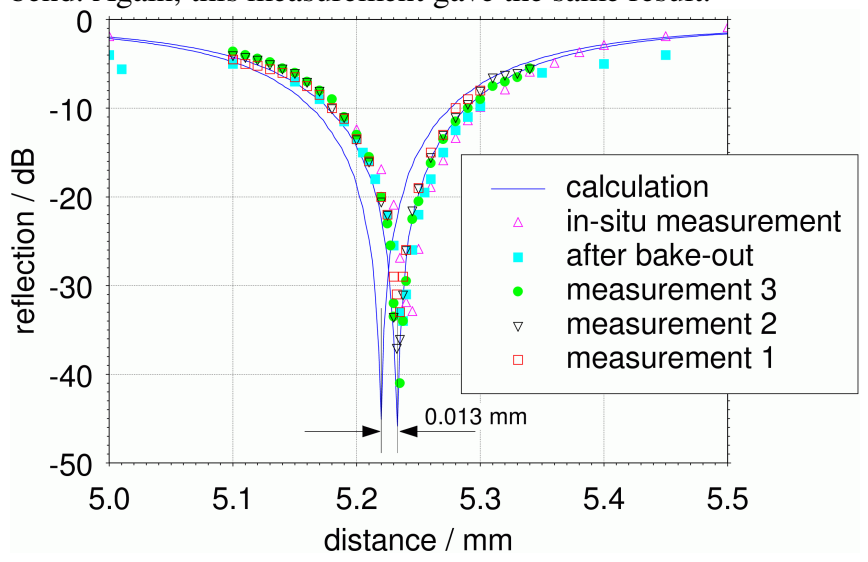

Fig. 2. Measured and calculated reflectivity of the CVD diamond double disc torus window at $\mathrm{f}=117.5 \mathrm{GHz}$ as a function of the distance between the two discs.

\section{LAUNCHER TESTS}

The launcher consists of a fixed focusing mirror and a plane mirror with a fast spindle drive and a push rod. Fast poloidal steering with up to $10 \% 100 \mathrm{~ms}$ has been achieved while the toroidal angle can be set in between pulses by rotating the steerable mirror around its own axis. First plasma experiments were done with pre-programmed launcher movements that were set by the ECRH control system before the discharge. For future feedback controlled operation of the ECRH system it is necessary to leave the control of the poloidal launcher movement during a plasma discharge with the main discharge control system (DCS) of ASDEX Upgrade. Fig.3 shows the first test of a launcher movement controlled by the DCS. The dashed line shows the desired position as required by the discharge program, the solid line gives the response of the launcher measured at the push rod. As can be seen from Fig.3 the launcher follows closely the path given by the control 
signal. The total poloidal angular sweep in this example was $8.5^{\circ}$.

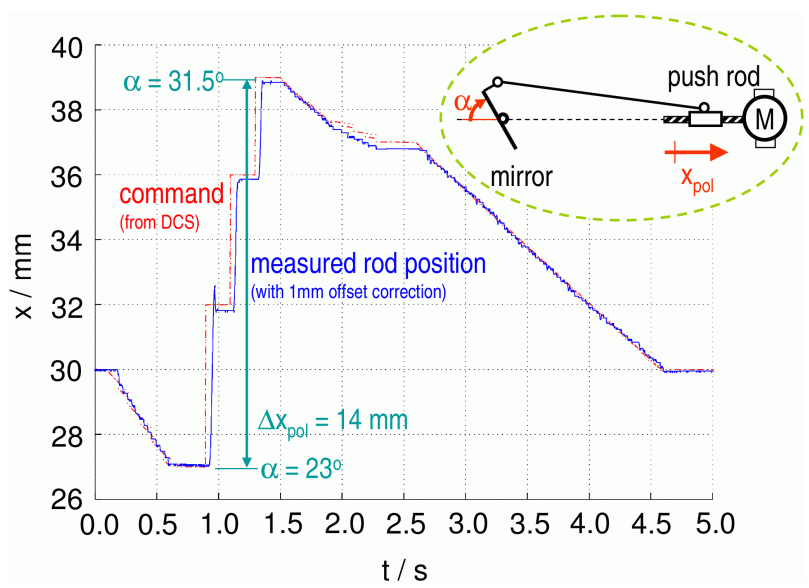

Fig.3. Poloidal ECRH launcher movement during a plasma discharge controlled by the DCS.

\section{EXPERIMENTAL RESULTS}

\section{A. OPERATION WITH W-WALLS}

Since early 2007 ASDEX Upgrade operates with fully tungsten covered plasma facing components in order to study this material for its use in future fusion reactors, which cannot cope with the high erosion rate and Tritium co-deposition found for carbon walls, which are in use in most of today's fusion experiments. The relative concentration of $\mathrm{W}$ in the plasma must be much lower $\left(<10^{-4}\right)$ than for $\mathrm{C}\left(<10^{-2}\right)$ since the radiation losses per ion are much larger. The operation of ASDEX Upgrade since 2007 showed that well confined Hmodes can be obtained with pure $\mathrm{W}$ surfaces, even without using boronization for wall conditioning [8]. For the pure Wsurfaces, i.e. without boronization, stable H-mode operation using only NBI as additional heating requires a high level of gas puff, resulting in moderate pedestal temperatures and high ELM frequency, both favorable for a low rate of $\mathrm{W}$ erosion. The usage of central ECRH reduces the central peaking of the tungsten concentration and allows to access higher pedestal temperatures. In figure 4 a plasma is shown, which is unstable without central ECRH, due to an uncontrollable increase of the central $\mathrm{W}$ concentration and the corresponding core radiation. The deposition of the ECRH is varied to find out how central it has to be to suppress the accumulation of tungsten. On top three different launching conditions are shown as calculated with TORBEAM [9]. Condition A corresponds to the central heating known to suppress accumulation. In a first experiment the fast poloidal mirror of the new system was used to move the ECRH deposition upwards (figure 4, middle). At about 5.0 sec, corresponding to launching condition $\mathrm{B}$, the tungsten concentration in the plasma center increases and the stored energy drops due to a reduction in core temperature. A cross check of this observation was made using the toroidal magnetic field instead of the poloidal launcher angle to vary the ECRH deposition as shown in figure 4, bottom. At $5.5 \mathrm{sec}$ the plasma becomes unstable corresponding to launching condition $\mathrm{C}$. In both cases the plasma becomes unstable when the deposition is roughly at the same flux surface (dashed line). This flux surface is in the vicinity of the flux surface for which the safety factor q equals unity. Actually at least two mechanisms seem to cause the effect of the ECRH on the W accumulation which are both still under study using modulation experiments. A clear change in $(1,1)$ MHD activity and sawteeth is seen comparing launching conditions $\mathrm{A}$ and $\mathrm{B}$ indicating a direct effect of the MHD on the heavy impurities. It is well known that such MHD modes are sensitive to the location of the ECRH with respect to the $q=1$ surface [10]. If, for launching position $B$, the ECRH is switched off, the $\mathrm{W}$ influx occurs much faster. This indicates that the central ECRH has an additional effect on the impurity transport outside the $\mathrm{q}=1$ surface. This has previously been explained by an increased diffusivity due to an increased turbulence level due to the central heating [11].

\section{B. PLASMA BREAKDOWN}

In future machines like ITER the superconductivity of the transformer coil will limit the loop voltage severely. Additionally, most of the flux swing of the transformer will be used to raise the plasma current. The length of the current flattop can be increased significantly if the plasma conductivity in the ramp-up phase of the discharge can be increased. Therefore the use of ECRH for pre-ionization of the neutral gas and subsequent ECR heating during the current rise phase are discussed for ITER [12]. Several of such scenarios both at 105 and $140 \mathrm{GHz}$ have been successfully tested at ASDEX Upgrade [13]. The one that worked best was at the first harmonic O-mode $(\mathrm{O} 1)$ with an on-axis magnetic field of $3.2 \mathrm{~T}$ using the two-frequency gyrotron at $105 \mathrm{GHz}$. Fig.5 shows the corresponding data where the plasma breakdown happened almost immediately, given by the $\mathrm{D}_{\alpha}$ radiation after the ECRH with approximately $400 \mathrm{~kW}$ was switched on. The plasma current rise could be controlled such that it was almost linear. Additionally the second harmonic X-mode scenario [14] has been tested with $140 \mathrm{GHz}$ at $2.2 \mathrm{~T}$, which works as well, but the pre-ionization takes some $10 \mathrm{~ms}$ longer as compared to $\mathrm{O} 1$. These scenarios are fully in line with the $170 \mathrm{GHz}$ envisaged for the main ECRH system of ITER for full toroidal field (5.3 $\mathrm{T}, \mathrm{O} 1)$ and half toroidal field $(2.6 \mathrm{~T}, \mathrm{X} 2)$. This means that the $170 \mathrm{GHz}$ ITER gyrotrons can be used also for plasma breakdown whereas originally a $127.5 \mathrm{GHz}$ startup system consisting of 3 gyrotrons was planned [15]. 
A

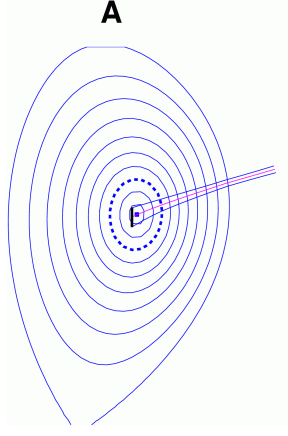

B

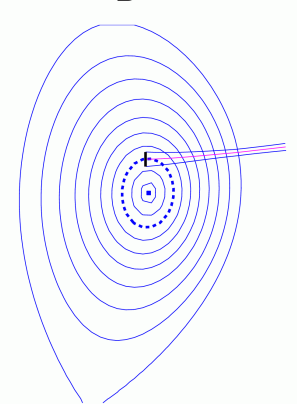

A
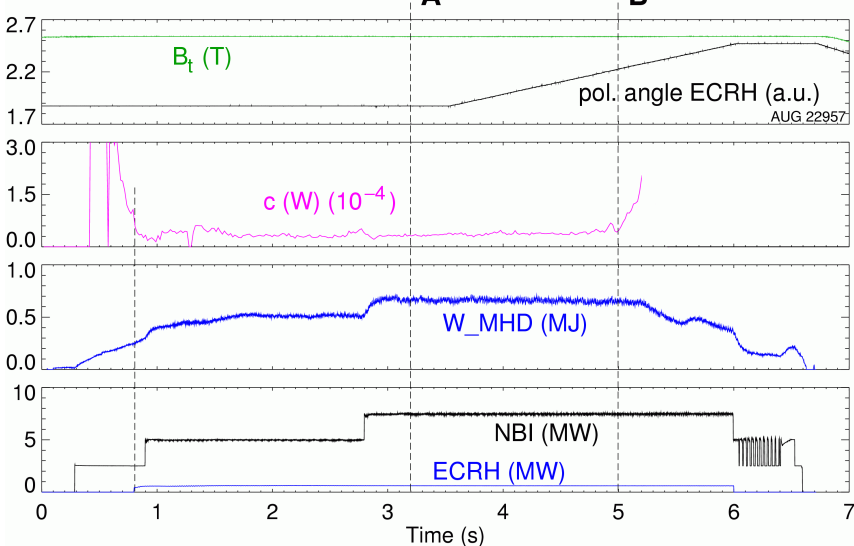

A
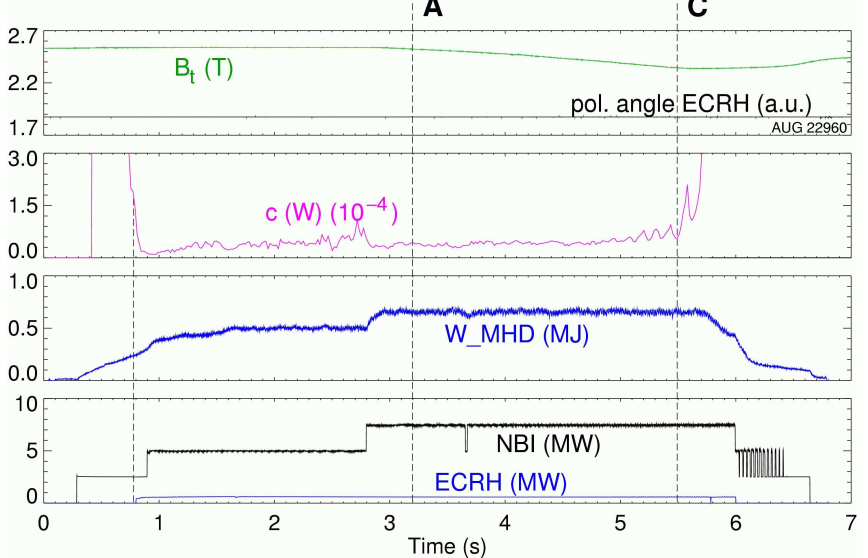

Fig.4. Variation of the ECRH deposition in the plasma center. Top: selected launching conditions, corresponding to the dashed time marks in the middle and bottom part. Middle: variation of the poloidal launching angle. Bottom: variation of the toroidal magnetic field. Plasma parameters at $3.2 \mathrm{~s}: \mathrm{I}_{\mathrm{p}}=1.0$ MA, $\mathrm{q}_{95}=4.8, \mathrm{n} / \mathrm{n}_{\mathrm{GW}}=0.65, \mathrm{H} 98(\mathrm{y}, 2)=0.95$. The ECRH power coupled to the plasma is $600 \mathrm{~kW}$.

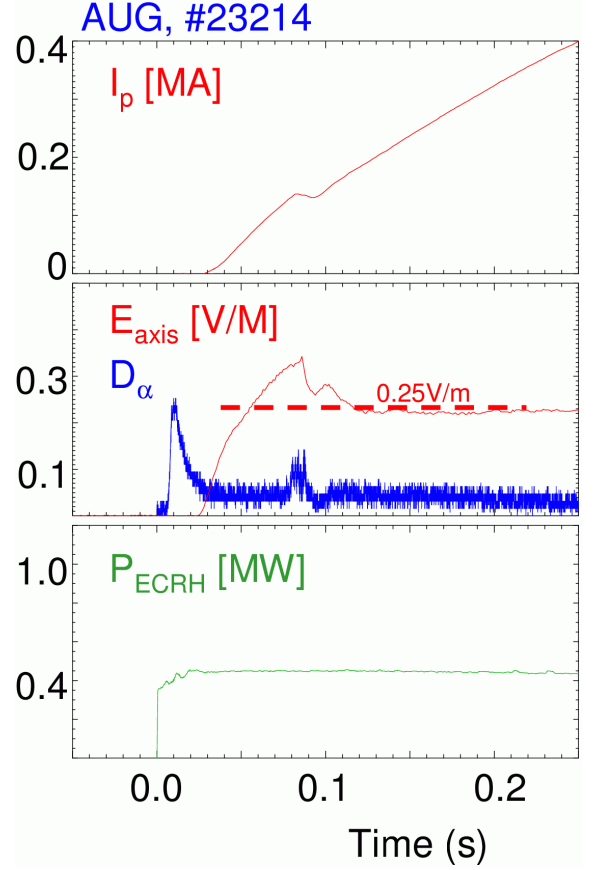

Fig.5. Plasma breakdown in ASDEX Upgrade using ECRH at $105 \mathrm{GHz}, 3.2 \mathrm{~T}$ corresponding to first harmonic O-mode.

\section{REFERENCES}

[1] F. Leuterer et al., "Operation Experience with the ASDEX Upgrade ECRH system", ECE-15.

[2] D. Wagner et al., "Present Status of the New Multifrequency ECRH System for ASDEX Upgrade", IEEE Trans. Plasma Science 36, 324282331, April 2008.

[3] G.G. Denisov et al., "Development in Russia of high power gyrotrons for Fusion", Nuclear Fusion, 48, 054007, May 2008.

[4] H. Zohm and M. Thumm, "On the use of step-tunable gyrotrons in ITER", J. of Phys: Conf. Series, 25, 274-282, May 2005.

[5] A. Manini et al., "Development of a feedback system to control MHD instabilities in ASDEX Upgrade", Fusion Eng. And Design, 82, 9951001, October 2007.

[6] D. Wagner and F. Leuterer, " Broadband Polarizers for High-Power Multi-Frequency ECRH Systems", Int. J. on Infrared and Millimeter Waves, 26, 163-172, February 2005.

[7] R. Heidinger et al., "Development of high power window prototypes for ECH\&CD launchers", Fusion Eng. And Design, 82, 693-699, October 2007.

[8] R. Neu et al., "Plasma wall interaction and its iplication in an all tungsten divertor tokamak", Plasma Phys. Controll. Fusion, 49, B49B70, October 2007.

[9] E. Poli et al., "EC beam tracing in fusion plasmas", Fusion Eng. Design, 53, 9-21, January 2001.

[10] A. Manini et al., "', Proc. $14^{\text {th }}$ Loint Workshop on ECE and ECRH, Santorini, Greece, Heliotopos Conference Ltd., Athens, 2006.

[11] R. Dux et al., submitted to Journ. Nucl. Materials.

[12] ITER Technical Basis 2002, ITER EDA Documentation Series No. 24, (Vienna: IAEA).

[13] G. Sips, Nuclear Fusion, to be published.

[14] G.L. Jackson, "Second harmonic electron cyclotron pre-ionization in the DIII-D tokamak", Nuclear Fusion, 47, 257-263, March 2007.

[15] N. Kobayashi et al., "Design of Electron Cyclotron Heating and Current Drive System of ITER", Proc. $17^{\text {th }}$ Top Conf. on Radio Freq. Power in Plasmas, AIP Conf. Proc. 933, 413-416, 2007. 